Revue internationale de l'économie sociale

Recma

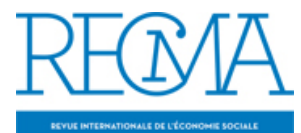

\title{
Théories, pratiques et droit : des tensions fécondes
}

\section{Jean-François Draperi}

Numéro 330, octobre 2013

La coopération : théories, pratiques et droit

Cooperative: Theory, Practice and Law

URI : https://id.erudit.org/iderudit/1019451ar

DOI : https://doi.org/10.7202/1019451ar

Aller au sommaire du numéro

Éditeur(s)

Association Recma

ISSN

1626-1682 (imprimé)

2261-2599 (numérique)

Découvrir la revue

Citer ce document

Draperi, J.-F. (2013). Théories, pratiques et droit : des tensions fécondes. Revue internationale de l'économie sociale, (330), 4-6.

https://doi.org/10.7202/1019451ar d'utilisation que vous pouvez consulter en ligne.

https://apropos.erudit.org/fr/usagers/politique-dutilisation/ 


\section{THÉORIES, PRATIQUES ET DROIT: DES TENSIONS FÉCONDES}

Ce numéro 330 de la Recma propose un dossier sur « La coopération : théories, pratiques et droit » comprenant quatre articles.

Dans sa contribution sur «Les fondements théoriques des coopératives de crédit », Nazik Beishenaly se penche sur les rôles politique et économique des coopératives de crédit, à partir d'une approche historique de la pensée coopérative.

Après avoir introduit aux principaux textes de Marx sur les coopératives de production, Hervé Defalvard montre toute l'actualité d'une analyse de la valeur centrée sur le travail. Il aborde à partir de cette question fondamentale de la valeur plusieurs problèmes essentiels pour la coopérative, comme le profit, la concurrence, la rémunération.

Abordant une évolution remarquable de la créativité coopérative, MarieChristine Barbot-Grizzo, France Huntzinger et Thierry Jolivet étudient la transmission de PME saines en Scop et soulignent l'importance de la relation de confiance qui se noue entre acteurs économiques et conseillers coopératifs.

Enfin, Willy Tadjudje analyse de façon critique le rôle de l'Organisation pour l'harmonisation en Afrique du droit des affaires (Ohada) dans la normalisation des règles coopératives d'épargne et de crédit.

Hors dossier, on lira un article de Patrick Valéau, Hassen Parak et Pierre Louart sur "Les équipes associatives, entre rationalité en valeur et entrepreneuriat social». Les auteurs y abordent la confrontation entre l'entrepreneuriat social et le fonctionnement associatif. Au terme d'une fine description de terrain, ils soulignent les difficultés d'articulation tout en montrant la possibilité d'aboutir à des mutations organisationnelles.

Enfin, dans la rubrique « Varia », Laurent Bisault revisite le périmètre coopératif: question difficile mais essentielle de la mesure des coopératives au sein de l'économie sociale et solidaire.

\section{UNE CONFRONTATION HEURISTIQUE ENTRE THÉORIES ET PRATIQUES}

L'essor constant des travaux conceptuels sur l'ESS à partir de diverses disciplines poursuivant chacune leur propre projet théorique pose plusieurs questions au praticien de l'ESS. Celui-ci se trouve confronté à des conceptions nouvelles, à un vocabulaire nouveau, proposant une interprétation théorique différente de la sienne. Cet apport l'oblige à réfléchir à la portée des théories qui lui sont proposées, à confronter celles-ci à son approche personnelle et, parfois, à effectuer un déplacement susceptible de questionner ses pratiques et leur rapport à l'éthique. Cette question est d'autant plus compliquée qu'elle se double d'un questionnement politique: l'impossibilité de la neutralité du concept au regard des valeurs suscite, audelà du débat théorique, des enjeux politiques qui occasionnent des actions de communication ou de lobbying. Et l'on sait que toutes les organisations et 
tous les acteurs institutionnels produisant des connaissances, les valorisant ou les transmettant ne communiquent pas avec la même puissance et le même bonheur. Or, sans nier le caractère heuristique et indispensable de la confrontation aux théories " exogènes », la majorité des praticiens, ainsi que de nombreux enseignants et chercheurs, sont également attachés à une théorisation des pratiques à partir des conceptions des praticiens eux-mêmes. D'où la proposition suivante: si nous pensons que l'ESS est susceptible de produire un apport original à la pensée contemporaine ${ }^{(1)}$, il faut avoir l'audace de faire vivre les concepts qu'elle a forgés, de les confronter à de nouveaux plutôt que d'adopter ceux-ci sous prétexte qu'ils viennent d'être produits. Pourquoi faudrait-il substituer le profit à l'excédent de gestion, le business plan (fût-il social) au projet d'activités, remplacer l'usager ou le bénéficiaire par le client, la recherche de fonds par le fundraising, dire que les réserves impartageables et inaliénables sont dépassées, ignorer la double qualité ou l'a-capitalisme? Abandonner ces concepts, c'est assurément nier la force d'invention de l'ESS et contribuer à la banaliser.

En effet, l'adoption de nouveaux termes généralement issus des pratiques capitalistes, et assez souvent du capitalisme financier, autorise également des entreprises classiques à revendiquer leur appartenance à l'ESS, ce qui témoigne d'ailleurs d'un retournement de tendance étonnant: les coopératives seraient désuètes, les associations mal gérées et les mutuelles dépassées, mais l'appartenance à l'ESS est intéressante. Ceux qui souhaitent que les sociétés de capitaux puissent être reconnues comme appartenant à l'ESS soulignent que des sociétés de capitaux peuvent être aussi éthiques que des entreprises de l'ESS.

\section{L'INÉPUISABLE QUESTION DES STATUTS}

C'est tout à fait vrai, à n'en pas douter, mais ajoutons que c'est aussi un tour de passe-passe, qui consiste à dire: puisque l'on peut être vertueux dans le cadre des sociétés de capitaux et ne pas l'être dans des entreprises d'ESS, la distinction entre les deux types n'a pas de sens. La démonstration s'appuie non sur la réalité de l'ensemble des deux types d'entreprises, mais sur l'observation d'exemples dont on n'interroge pas la représentativité. Ce raisonnement postule que des statuts d'entreprises d'ESS devraient obligatoirement engendrer des comportements vertueux. Cependant, en dehors de la théorie, en dehors de l'idéal-type, un tel statut est le propre d'une institution totalitaire.

C'est ainsi en reprochant à l'ESS de ne pas être parfaite que l'on conclut à la négation de l'intérêt de distinguer des types d'entreprises et que l'on s'interdit toute analyse sur la réalité des différences entre l'ESS et l'économie capitaliste, sans même avoir pris la peine d'observer l'ensemble de la réalité observable. C'est en ce sens que l'on peut parler de tour de passe-passe.

Or, le dessein poursuivi par l'ESS n'est pas de concevoir l'entreprise idéale dont rêvent ses détracteurs, mais de définir des règles nouvelles qui permettent un fonctionnement meilleur de l'économie et de l'entreprise. Sous cet angle, les statuts constituent des garde-fous et plus fondamentalement une conquête 
sociale: celle de rendre possible une entreprise fondée non sur la réunion de capitaux, mais sur le groupement de personnes. Les statuts ne garantissent nullement la vertu, et d'ailleurs jamais personne ne l'a prétendu: au contraire, comme le disait Charles Gide il y a un siècle, les statuts sont nécessaires précisément pour ne pas dépendre de la trop incertaine vertu des hommes.

Mais si l'on veut placer son capital pour le rémunérer, mieux vaut aller dans une société de capitaux plutôt que dans une coopérative, et si l'on veut participer au pouvoir en tant que salarié, mieux vaut être dans une Scop que dans une SA. Les statuts ne sont pas neutres. Ils sont nécessaires tout en étant insuffisants.

\section{LES ÉCARTS DE SALAIRES: UNE MESURE IMPOSSIBLE?}

Autre débat animé: les écarts de salaires. Au terme de son analyse sur la valeur, Hervé Defalvard rappelle que le Ceges propose des écarts de salaires allant de 1 à 20 , le Mouves des écarts allant de 1 à 10 et le secteur de l'insertion par l'activité économique, des écarts allant de 1 à 5 . L'écart entre les écarts proposés par le Ceges et le secteur de l'insertion par l'activité économique questionne. Parle-t-on de la même chose? Et pourquoi personne ne propose la stricte égalité des salaires? Impossible, certes, mais de même qu'est impossible un écart allant de 1 à 5 dans certaines entreprises... alors qu’il est déjà exagéré dans d'autres. Autant cette question des écarts de salaires est importante, autant elle ne peut pas être posée, ni identiquement à l'ESS dans son ensemble, ni uniquement à l'ESS.

Plus encore, elle ne peut pas être posée en ne considérant que l'entreprise. Par exemple, les coopératives vinicoles champenoises ont dû payer, cher, des chefs de cave qui jusqu'alors opéraient dans le négoce, lorsqu'elles ont voulu entreprendre la champagnisation. Soit elles augmentaient considérablement l'écart des salaires afin de produire un champagne de qualité, soit elles abandonnaient cette ambition. Dans ce cas, des milliers de petits viticulteurs auraient disparu et laissé la place aux multinationales que sont les plus grands négociants. La question ne s'est d'ailleurs pas posée: il s'agissait de la survie des exploitations familiales.

\section{DES RECHERCHES ET DES ÉTUDES}

Pour évaluer les écarts de salaires, de même que pour mesurer les spécificités des groupements de personnes vis-à-vis des sociétés de capitaux, il semble donc indispensable de comparer les entreprises d'ESS et les entreprises capitalistes comparables (en particulier du point de vue du secteur d'activité, du rapport au marché et de la taille) et de comparer les produits, la communication, les conditions de travail, les écarts de salaires, la destination des profits, l'activité de lobbying, etc. Il est également essentiel de considérer les effets induits de la présence d'une entreprise d'ESS sur un marché ou sur un territoire.

Raisons pour lesquelles la Recma tient à publier des recherches théoriques, qui nourrissent des débats contradictoires, mais tout autant des études de terrain, qui ne cessent de révéler la riche complexité de sa réalité... et qui permettent d'apprécier la portée des recherches du point de vue de l'action. 